\title{
In Saccharomyces cerevisiae deletion of phosphoglucose isomerase can be suppressed by increased activities of enzymes of the hexose monophosphate pathway
}

\author{
J. Richard Dickinson, ${ }^{1}$ Michael A. Sobanski ${ }^{1}$ and Michael J. E. Hewlins ${ }^{2}$
}

Author for correspondence: J. Richard Dickinson. Tel: +44 1222874000 ext. 5762. Fax: +441222874305.

\footnotetext{
1 School of Pure and Applied Biology, University of Wales College of Cardiff, PO Box 915, Cardiff CF1 3TL, UK

2 Department of Chemistry, University of Wales College of Cardiff, PO Box 912, Cardiff CF1 3TB, UK
}

\begin{abstract}
Saccharomyces cerevisiae mutants defective in the structural gene PGI1 lack phosphoglucose isomerase and hence cannot grow on glucose. Spontaneous mutants were isolated by selecting for the regained ability to grow on YEPD (yeast extract/peptone/glucose). Three complementation groups called spg29-31 (suppressor of pgi1 $\Delta$ ) were identified. The metabolism of $\left[2-{ }^{13} \mathrm{C}\right.$ ]glucose was studied by ${ }^{13} \mathrm{C}$ NMR spectroscopy. This led to the conclusion that in a spg29 mutant suppression of the glycolytic defect was achieved by increased carbon flux through the hexose monophosphate pathway. The specific activities of enzymes of the hexose monophosphate pathway (except glucose-6-phosphate dehydrogenase) and NAD- and NADP-dependent glutamate dehydrogenase were increased in the bypass mutant.
\end{abstract}

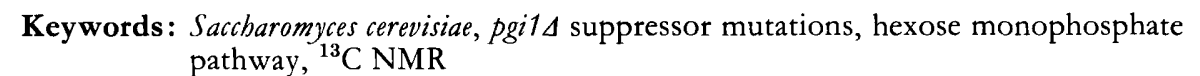

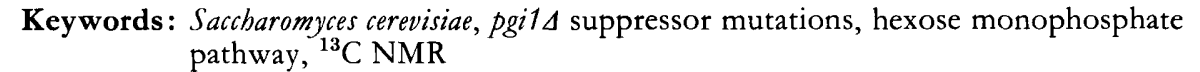

\section{INTRODUCTION}

Phosphoglucose isomerase is the enzyme which catalyses the interconversion of glucose 6-phosphate and fructose 6-phosphate. Thus, it is both the second step of the glycolytic sequence and the last step of gluconeogenesis. Mutants of Saccharomyces cerevisiae defective in the structural gene (PGI1) for phosphoglucose isomerase have been described by many authors (Maitra, 1971; Herrera \& Pascual, 1978; Clifton et al., 1978; Ciriacy \& Breitenbach, 1979; Aguilera, 1986). All such pgi1 mutants cannot grow on glucose, the assumption being that in S. cerevisiae flux through the hexose monophosphate pathway is inadequate to support growth. It is reckoned that the hexose monophosphate pathway is only capable of $8 \%$ of the carbon flux that occurs during growth on glucose (Bruinenberg et al., 1986). In this respect $S$. cerevisiae is different from both Escherichia coli and Kluyveromyces lactis, in which mutants lacking phosphoglucose isomerase can still grow on glucose (Vinopal et al., 1975; Goffrini et al., 1991).

One way of trying to understand all aspects of the phenotype of pgi1 mutants has been the isolation of extragenic suppressor mutations which confer upon pgi1 mutants the ability to grow on glucose. Aguilera (1987) described spg1 (suppressor of pgi14) mutations which restored growth on glucose and resulted in obligately high levels of mitochondrial respiration and no ethanol formation. Gamo et al. (1993) described rgll and $\mathrm{rgl}$ (resistance to glucose) mutations from which it was also presumed that glucose may be channelled through the hexose monophosphate pathway to respiration. Recently, Boles et al. (1993) showed that overexpression of GDH2, which encodes the NAD-dependent glutamate dehydrogenase, can suppress the growth defect on glucose caused by pgitd mutations. The explanation for this is that overexpression of NAD-dependent glutamate dehydrogenase causes a cycle of metabolic interconversion between 2-oxoglutarate and glutamate in which the anabolic reaction results in the conversion of 2-oxoglutarate to glutamate with concomitant conversion of NADPH to NADP, whilst the catabolic reaction results in the formation of 2-oxoglutarate from glutamate with conversion of NAD to NADH. Hence, the rapid depletion of NADP which would otherwise result from overuse of glucose-6-phosphate dehydrogenase (if flux via the hexose monophosphate pathway was increased in pgi1 1 bypass mutants) is avoided and the extra NADH which is formed can be oxidized by the electron transport chain. This also explains the high obligatory respiration observed in pgi1 bypass mutants.

Boles et al. (1993) also showed that deletion of the ZWF1 


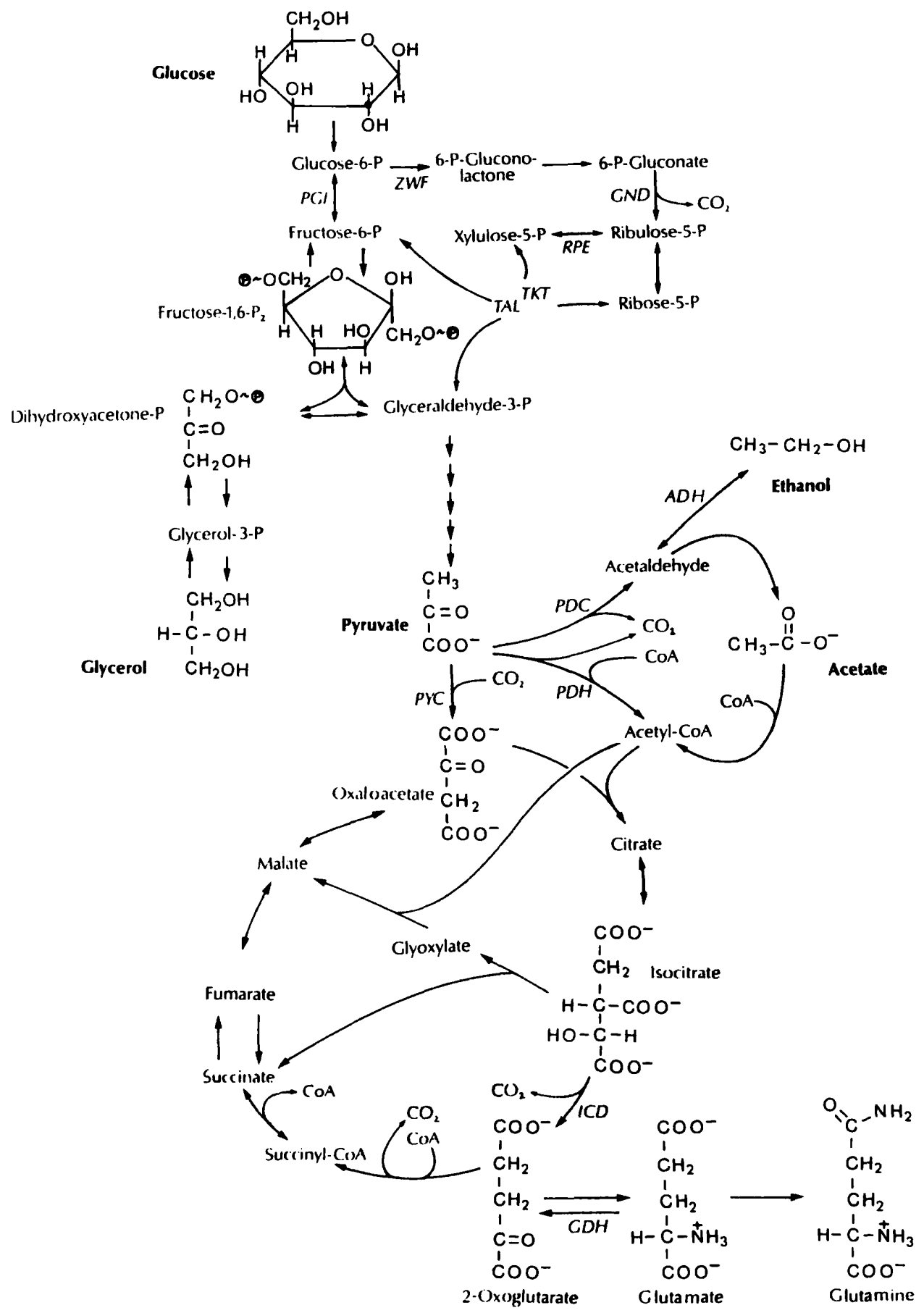

Fig. 1. The pathways of catabolism of glucose in S. cerevisiae. The figure is redrawn from a version by Fraenkel (1982). The structures of all of the intermediates and products discussed in the text are given. Only the $\beta$ anomer of the substrate $\mathrm{D}$-glucose is drawn. The ${ }^{13} \mathrm{C}$ label at $\mathrm{C}-2$ of glucose is marked by (0). Enzymes which are specifically mentioned are identified by three-letter acronyms as follows: ZWF, glucose-6-phosphate dehydrogenase; PGI, phosphoglucose isomerase; GND, 6-phosphogluconate dehydrogenase; RPE, ribulose-5-phosphate 3-epimerase; TAL, transaldolase; PDC, pyruvate decarboxylase; $A D H$, alcohol dehydrogenase; $P D H$, pyruvate dehydrogenase; $I C D$, isocitrate dehydrogenase; $G D H$, glutamate dehydrogenase. Irreversible reactions are denoted by single-headed arrows. The presence of two arrows indicates a separate enzyme for each direction of a reaction. Reversible reactions are represented by bi-directional arrows (except, for simplicity, the four reversible reactions 'below' glyceraldehyde-3-phosphate). 
gene (which encodes glucose-6-phosphate dehydrogenase) blocks suppression of a pgi1 $\Delta$ mutation. This is also highly indicative of the importance of the hexose monophosphate pathway, but it must be stressed that, to date, none of the studies have proved that the suppression of a pgi1 mutation actually involves a metabolic bypass in which glucose is catabolized by means of increased flux via the hexose monophosphate pathway; i.e. the flow of carbon from substrate to product(s) has not been demonstrated. One could postulate three possible metabolic routes. Firstly, increased flux via the hexose monophosphate pathway. Secondly, use of the glycolytic sequence involving an isoenzyme of phosphoglucose isomerase encoded by a gene which is normally cryptic. This is not without precedent in S. cerevisiae: for example $A D H 4$ (encoding an isoenzyme of ethanol dehydrogenase) was discovered in $a d h 1$ adh 2 adb 3 triple mutants (Paquin \& Williamson, 1986). Thirdly, the use of a hitherto-unknown pathway of glucose catabolism. This concept has also been suggested previously to explain the bypass of $p f k 1 p f k 2$ double mutants which lack phosphofructokinase (Breitenbach-Schmitt $e t$ al., 1984). ${ }^{13} \mathrm{C}$ nuclear magnetic resonance (NMR) spectroscopy is a very good technique for metabolic studies (London, 1988; Davies \& Brindle, 1992); hence it was decided to use ${ }^{13} \mathrm{C}$ labelled glucose to analyse the patterns of metabolism by ${ }^{13} \mathrm{C}$ NMR in suppressed pgi1 14 mutants. Providing yeast with $\left[2-{ }^{13} \mathrm{C}\right]$ glucose allows one to discriminate between the known pathways as described below.

Use of the hexose monophosphate pathway will give rise to fructose 1,6-bisphosphate with label at C-1 and C-3. Subsequent cleavage by aldolase would yield dihydroxyacetone phosphate labelled at C-1 and C-3, which would be metabolized by the conventional glycolytic sequence to produce pyruvate labelled at C-3 and C-1, respectively. This labelled pyruvate has three possible fates, the first being conversion to ethanol (labelled at $\mathrm{C}-2$ ) and ${ }^{13} \mathrm{CO}_{2}$ via pyruvate decarboxylase and ethanol dehydrogenase (Fig. 1). Since it is common for ethanolic fermentation to be accompanied by the formation of some glycerol (to preserve the NAD/NADH balance) one would also expect to see glycerol labelled at C-1 and C-3 which was derived from the C-1,3-labelled dihydroxyacetone phosphate (Fig. 1). A second possible fate of the pyruvate would be via pyruvate dehydrogenase, which would result in loss of ${ }^{13} \mathrm{CO}_{2}$ and formation of $\left[2-{ }^{13} \mathrm{C}\right]$ acetate. Subsequent metabolism of the $\left[2-{ }^{13} \mathrm{C}\right]$ acetate via the tricarboxylic acid and/or glyoxylate cycles would give predictable labelling patterns as described previously (Dickinson et al., 1983; Dickinson \& Hewlins, 1991) when the fate of $\left[2-{ }^{13} \mathrm{C}\right]$ acetate was analysed by ${ }^{13} \mathrm{C}$ NMR. The third possible route for metabolism of some pyruvate would be via pyruvate carboxylase to 1,3-labelled oxaloacetate. The ${ }^{13} \mathrm{C}$ at $\mathrm{C}-1$ of oxaloacetate would be lost as ${ }^{13} \mathrm{CO}_{2}$ at the isocitrate dehydrogenase step of the tricarboxylic acid cycle and the remaining label would result in glutamate labelled only at C-2 - an isotopomer that would be easy to identify, but has never been seen in yeast. Metabolism of isocitrate via the glyoxylate cycle would give readily identifiable intermediates.
The expression of a previously cryptic gene resulting in a new phosphoglucose isomerase activity would give catabolism of $\left[2-{ }^{13} \mathrm{C}\right]$ glucose via a completely reinstated glycolytic sequence. This would be expected to produce $\left[2-{ }^{13} \mathrm{C}\right]$ pyruvate and hence ethanol labelled at $\mathrm{C}-1$ along with some $\left[2{ }^{13} \mathrm{C}\right]$ glycerol (Fig. 1). Oxidative decarboxylation of this $\left[2{ }^{13} \mathrm{C}\right]$ pyruvate via pyruvate dehydrogenase would give $\left[1-{ }^{13} \mathrm{C}\right]$ acetate, which could enter the tricarboxylic acid cycle to yield glutamate labelled at $\mathrm{C}-1$ on the first turn of the cycle and thence at $\mathrm{C}-5$ on the second turn. The labelling of glutamate at $\mathrm{C}-1$ and $\mathrm{C}-5$ would yield a very characteristic spectrum. Metabolism of the $\left[1-{ }^{13} \mathrm{C}\right]$ acetate to $\left[6^{-13} \mathrm{C}\right]$ isocitrate would give rise via the glyoxylate cycle to glutamate labelled only at $\mathrm{C}-1$ on each successive turn of that cycle. The conversion of $[2-$ $\left.{ }^{13} \mathrm{C}\right]$ pyruvate to $\left[2-{ }^{13} \mathrm{C}\right]$ oxaloacetate by pyruvate carboxylase would give rise to glutamate labelled at $\mathrm{C}-3$ on the first turn of the tricarboxylic acid cycle, 2,3- on the second turn and 1,2,3- on the third and subsequent turns of that cycle. This would also be a unique labelling pattern, which, even if it was combined with the formation of $1-$, and 1,5-labelled glutamate, would still be very different from patterns which have been recorded previously. Finally, conversion of any $\left[2{ }^{13} \mathrm{C}\right]$ oxaloacetate to $\left[3{ }^{13} \mathrm{C}\right]$ isocitrate and subsequent intervention of the glyoxylate cycle would yield glutamate labelled only at $\mathrm{C}-2$ and $\mathrm{C}-3$. Hence, the catabolism of $\left[2-{ }^{13} \mathrm{C}\right]$ glucose via all known metabolic routes (Fig. 1) produces predictable labelling patterns in intermediates whose constituent carbon atoms have well-defined resonances in the NMR spectrum. With this knowledge we embarked upon an NMR analysis of the metabolism of glucose in a pgi14 mutant carrying a spg 29 suppressor mutation which allows growth on glucose.

\section{METHODS}

Media, strains and genetic methods. Cultural conditions and complex growth media have been described previously (Dickinson, 1991). Complex media contained, per litre: yeast extract $(10 \mathrm{~g})$, bacteriological peptone $(20 \mathrm{~g})$, adenine $(0 \cdot 1 \mathrm{~g})$, uracil $(0 \cdot 1 \mathrm{~g})$ and the specified carbon source (YEPD, $20 \mathrm{~g}$ Dglucose; YEPF, $20 \mathrm{~g}$ D-fructose). Minimal medium with fructose $(2 \%, \mathrm{w} / \mathrm{v})$ as major carbon source was supplemented with $0 \cdot 1 \%(\mathrm{w} / \mathrm{v})$ glucose. Standard genetic techniques were used for mating, sporulation and dissection (Sherman, 1975; Mortimer \& Hawthorne, 1975). S. cerevisiae strain AAG2 (MATa ura3 his4 can pgi1 $125:$ : LEU2) was obtained from A. Aguilera (University of Seville, Spain). Strain S9 (MATa ura3 bis4 can pgi1425:: LEU2 spg29-1) was derived by crossing E9 (obtained as a spontaneous revertant of AAG2 as described below) to strain 329 (MATa ade8) and repeated backcrossing of appropriate $M A T$ a segregants to AAG2. Strain OH1 ( $M A T$ a ura 3 bis4 can) is a wild-type (i.e. PGI1) haploid derived from a cross between 329 and AAG2. Strains 26.1A (MATa ade 1 ade 2 bis7 pgi1-1) and 26.6B (MATa ura1 pgi1-1) were constructed from mutant 9520b/TIC (MAT $\alpha$ pgi1-1) (Maitra, 1971), which was provided by P. K. Maitra (Tata Institute of Fundamental Research, Bombay, India).

Isolation of mutants carrying suppressors of pgi1s mutations. A $100 \mathrm{ml}$ batch of strain AAG2 was grown overnight in YEPF to $\mathrm{OD}_{600} 1 \cdot 00$. The cells were then harvested by 
centrifugation and resuspended in $100 \mathrm{ml}$ YEPD. The culture was divided into 10 separate aliquots, each of which was incubated in a $100 \mathrm{ml}$ conical flask at $30^{\circ} \mathrm{C}$ for $6 \mathrm{~d}$. After this time $0.1 \mathrm{ml}$ samples were removed from each small culture and plated directly onto YEPD. The YEPD plates were inspected after 3,5 and $7 \mathrm{~d}$. Colonies which had appeared at each time and were large enough to handle were transferred to YEPF, incubated for 4-5 d and then replica-plated to YEPF and YEPD. Clones which were able to grow well on YEPD when re-tested were retained for further analysis.

NMR analyses. For in vivo NMR analysis cells of S9 were grown in YEPD to $\mathrm{OD}_{600} 4.0$ (late exponential phase for this strain), harvested by centrifugation, resuspended in $2 \mathrm{ml} 50 \mathrm{mM}$ potassium phosphate buffer $\mathrm{pH} 6.0$ containing $30 \%(\mathrm{v} / \mathrm{v})^{2} \mathrm{H}_{2} \mathrm{O}$ and transferred to a $10 \mathrm{~mm}$ NMR tube with a gassing system which provides both aeration and mixing (described by Lloyd $e t$ al., 1993). Then $70 \mathrm{mg}\left[2-{ }^{13} \mathrm{C}\right]$ glucose (99 atom \% enrichment) was added and ${ }^{13} \mathrm{C}$ data were collected in blocks of 500 accumulations (each of $8 \mathrm{~K}$ data points over $22000 \mathrm{~Hz}$ ) with an acquisition time of $0 \cdot 18 \mathrm{~s}$ and a $1 \mathrm{~s}$ delay between pulses using a Bruker WM360 spectrometer operating at $90.5 \mathrm{MHz}$. Highpower ${ }^{1} \mathrm{H}$-decoupling was used during acquisition, with lowpower decoupling during delays: preliminary experiments had determined the correct settings to effect proton decoupling with sufficient dielectric heating to maintain a temperature of $30{ }^{\circ} \mathrm{C} \pm 1{ }^{\circ} \mathrm{C}$.

Metabolic analyses were also done on cells which had been grown to $\mathrm{OD}_{600} 4.0$ in YEPD, harvested by centrifugation and resuspended in YEP containing $\left[2-{ }^{13} \mathrm{C}\right]$ glucose. After $150 \mathrm{~min}$ incubation at $30{ }^{\circ} \mathrm{C}$ cells were harvested and perchloric acid extracts prepared for ${ }^{13} \mathrm{C}$ NMR analysis as described previously (Dickinson \& Hewlins, 1988, 1991). The spectrum was recorded for the solution in a $5 \mathrm{~mm}$ NMR tube, using $32 \mathrm{~K}$ data points over $22000 \mathrm{~Hz}$, with broad-band ${ }^{1} \mathrm{H}$-decoupling, and also by the DEPT method to determine the number of protons attached to each carbon signal. A two-dimensional ${ }^{13} \mathrm{C}-{ }^{1} \mathrm{H}$ chemical shift correlation spectrum was carried out using the XHCORRD routine in the standard Bruker NMR software. All chemical shifts are reported in p.p.m. relative to the signals $\left({ }^{1} \mathrm{H}\right.$ and ${ }^{13} \mathrm{C}$ as appropriate) from sodium 3-(trimethylsilyl)propane-1-sulphonate (assigned $\delta=0$ for both nuclei) measured in ${ }^{2} \mathrm{H}_{2} \mathrm{O}$ solution as the external standard.

Enzyme assays. Cells were harvested by centrifugation, resuspended in $50 \mathrm{mM}$ potassium phosphate buffer $\mathrm{pH} 7 \cdot 4$ containing $2 \mathrm{mM}$ EDTA and $2 \mathrm{mM}$ 2-mercaptoethanol and disrupted using a Braun homogenizer as described by Dickinson \& Williams (1986). Aliquots of this homogenate were used immediately as the source of enzyme. Phosphoglucose isomerase (EC 5.3.1.9), glucose-6-phosphate dehydrogenase (EC 1.1.1.49) and gluconate-6-phosphate dehydrogenase (EC 1.1.1.43) were assayed as described by Maitra \& Lobo (1971). Ribulose-5-phosphate 3-epimerase (EC 5.1.3.1) was assayed according to Williamson \& Wood (1966). Transaldolase (EC 2.2.1.2) was assayed by the method of Tchola \& Horecker (1966). NAD- and NADP-dependent glutamate dehydrogenases (EC 1.4.1.2 and 1.4.1.4) were both assayed in $0.1 \mathrm{M}$ imidazole buffer $\mathrm{pH} 7.9$ in a total volume of $1.8 \mathrm{ml}$. NADPdependent glutamate dehydrogenase was assayed by following NADPH disappearance when 2-oxoglutarate $(22.8 \mu \mathrm{mol})$ was converted to glutamate in the presence of ammonium acetate $(614 \mu \mathrm{mol})$ using $0.03 \mu \mathrm{mol} \mathrm{NADPH}$. NAD-dependent glutamate dehydrogenase was monitored by following the increase in absorbance at $340 \mathrm{~nm}$ due to $\mathrm{NADH}$ formation when glutamate $(25 \mu \mathrm{mol})$ was converted to 2-oxoglutarate using $0 \cdot 375 \mu \mathrm{mol}$ NAD.

\section{RESULTS}

\section{Isolation and genetic characterization of mutants carrying suppressors of pgi1 $\Delta$ mutations}

The spontaneous mutants which arose on YEPD after $3 \mathrm{~d}$ were called 'early mutants' and numbered E1-E33. The 'mid mutants' collected after $5 \mathrm{~d}$ were numbered M1M14 and the 'late mutants' were numbered L1-L29. There was no correlation with the original time of appearance of an individual mutant and its rate of proliferation when examined on YEPD plates or in YEPD liquid medium: i.e. there was a range of generation times amongst all three sets of mutants. However, all of the 'mid mutants' subsequently died over the next 4 weeks (irrespective of whether they were stored on YEPD or YEPF) before they had been characterized genetically. Nevertheless, the frequency of suppressor mutations would seem to be $10^{-7}-10^{-8}$. Sixteen of the remaining mutants which grew most rapidly on YEPD were mated on YEPF to strain 26.1A. The diploids which formed were selected on fructose minimal medium supplemented with $0.1 \%$ glucose. These diploids were then replicated onto YEPD: none of them grew on YEPD, showing that the suppressor mutations were all recessive. A series of outcrosses to suitable wild-type haploids allowed a subsequent complementation analysis, which indicated the existence of at least three distinct $s p g$ mutations. It seemed prudent to retain the terminology of Aguilera (1987) for such mutants rather than add a further acronym to an already crowded literature of phenotypes. However, to avoid the possibility of a future overlap of mutants from our different laboratories it was decided to call these three mutations spg29 to spg31. It was not possible to

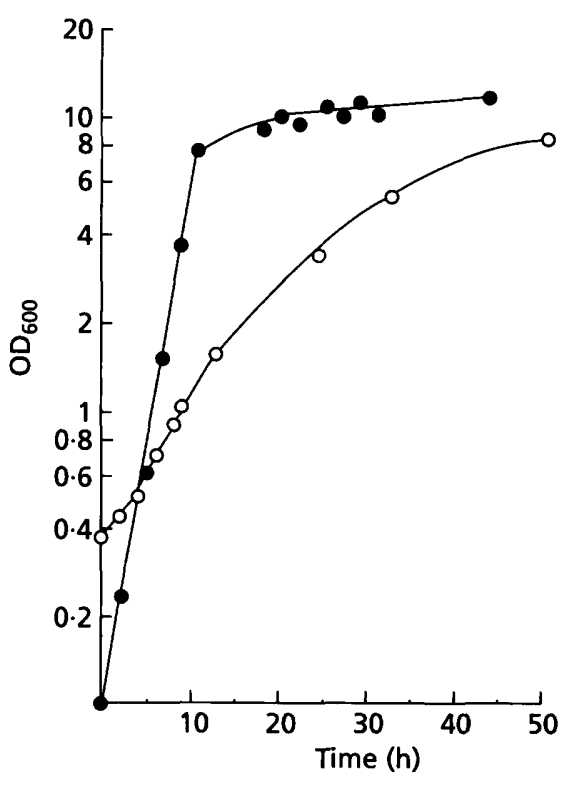

Fig. 2. Typical growth curves for $\$ 9$ (pgi1 $\Delta$ spg29-1; $O$ ) and wild-type haploid OH1 (PG/1; O) in YEPD liquid medium. Both strains were inoculated into fresh pre-warmed medium from starter cultures grown to exponential phase in YEPD. 


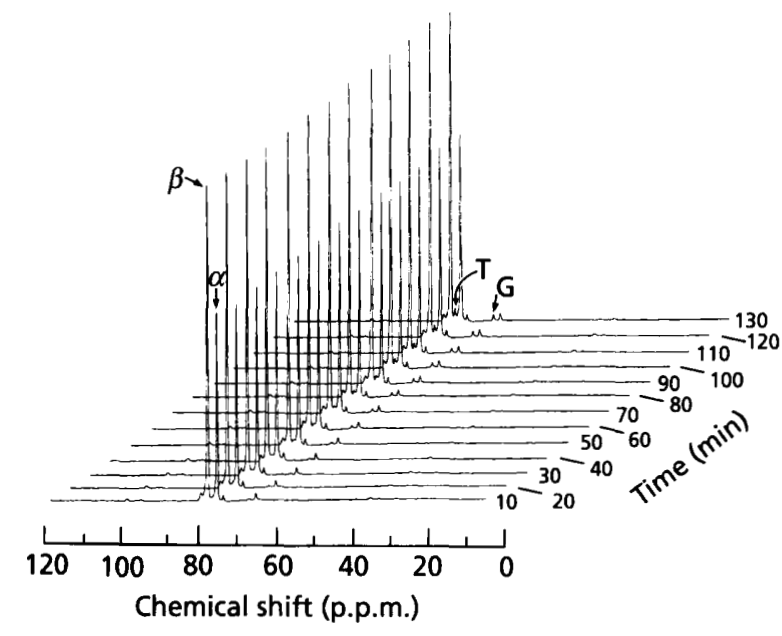

Fig. 3. Time course of utilization of $\left[2-{ }^{13} \mathrm{C}\right]$ glucose by strain 59 . In vivo time-elapsed proton decoupled NMR spectra are shown. $\alpha, \mathrm{C}-2$ of $\alpha$-D-glucose; $\beta, \mathrm{C}-2$ of $\beta$-D-glucose; T, C-2 of trehalose; G, C-1,3 glycerol.

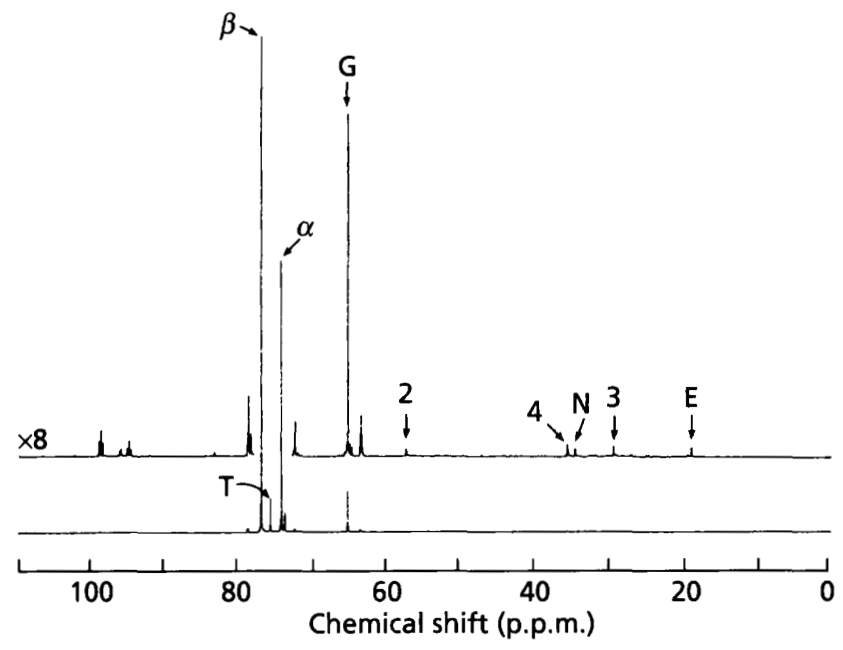

Fig. 4. ${ }^{13} \mathrm{C}$ NMR spectrum of a perchloric acid extract of strain 59 made after growth in YEP- $\left[2-{ }^{13} \mathrm{C}\right]$ glucose. E, C-2 of ethanol; 4, C-4 of glutamate; 3, C-3 of glutamate; 2, C-2 of glutamate; $\mathrm{N}, \mathrm{C}-4$ of glutamine; other resonances marked are as in Fig. 3.

definitely assign all of the mutants, mainly due to their different rates of proliferation on YEPD prior to mating. The spg29 mutation was represented by three alleles all resulting in very similar phenotypes, the most prominent of which was the most rapid growth (generation time of $335 \mathrm{~min}$ in exponential phase) in glucose (Fig. 2).

\section{${ }^{13} \mathrm{C}$ NMR analysis of metabolism in a pgi14 spg29 mutant}

In vivo $\mathrm{NMR}$ analysis of strain $\mathrm{S} 9$ showed that the major metabolic product derived from $\left[2-{ }^{13} \mathrm{C}\right]$ glucose was glycerol labelled at C-1 and C-3 (Fig. 3). This signal corresponding to glycerol (65.3 p.p.m.) increased with time as did the resonance corresponding to $\mathrm{C}-2$ of the storage compound trehalose ( 75.7 p.p.m.). Three other resonances can be seen in Fig. 3 (78.6 p.p.m., 72.4 p.p.m. and 63.5 p.p.m.); they are explained below. The in vivo experiment was not run for longer because although it could be seen (from comparison of the peak heights of the signals due to $\mathrm{C}-2$ of glucose) that very little glucose had been metabolized, the experiment had served its purpose, i.e. identification of the metabolic pathway by which the glucose was catabolized. Detailed isotopomer analysis is always better done on perchloric acid extracts because field inhomogeneity within the sample of in vivo experiments leads to signal broadness.

One possible criticism of the in vivo NMR study above is that the cells are in a non-growing situation because no nitrogen source was provided, although other workers actually strive to devise conditions of rigorous nongrowth for metabolic analysis (e.g. Benevolensky et al., 1994). However, in this case the criticism would seem to be irrelevant because analysis of an extract made from cells grown in YEP- $\left[2-{ }^{13} \mathrm{C}\right]$ glucose gave a similar result (Fig. 4). In addition, the expanded spectrum allows the identification of resonances at 18.9 p.p.m. (ethanol C-2), 29.4 p.p.m. (glutamate C-3), $35 \cdot 6$ p.p.m. (glutamate C-4) and 57.3 p.p.m. (glutamate C-2). Although small, the signal due to ethanol $\mathrm{C}-2$ is highly significant because it confirms that ${ }^{13} \mathrm{C}$ in this molecule has been derived via the hexose monophosphate pathway (as explained in the Introduction). The fact that the intensity of the signal for glutamate C-4 is significantly greater than that for C-3, which in turn is much greater than the intensity of the glutamate $\mathrm{C}-2$ resonance, is also important because these data indicate that glutamate is being labelled as predicted for metabolism of $\left[2-{ }^{13} \mathrm{C}\right]$ glucose around the hexose monophosphate pathway to yield pyruvate labelled at $\mathrm{C}-3$ and $\mathrm{C}-1$. This is decarboxylated to $\left[2-{ }^{13} \mathrm{C}\right]$ acetate, which then enters the tricarboxylic acid cycle. Indeed, it is just possible to observe the appearance of label in the C-4 position of glutamate first, followed by label at C-3 and $\mathrm{C}-2$, in the time-elapsed spectra in Fig. 3. The resonance at 34.6 p.p.m. is due to $\mathrm{C}-4$ of glutamine formed directly from glutamate. The multiplets at 98.6 p.p.m. and 94.8 p.p.m. are respectively C-1 of $\beta$-Dglucose and $\alpha$-D-glucose which are visible mainly due to natural abundance of ${ }^{13} \mathrm{C}$ at this position. The singlet (centre line)resonance in each case corresponds to glucose labelled only at $\mathrm{C}-1$; the doublets are due to interaction with the heavily labelled $\mathrm{C}-2$. Similarly, the resonance at 63.5 p.p.m. seen here and noted in Fig. 3 along with the resonances at 78.6 p.p.m. and 72.4 p.p.m. are due to glucose $\mathrm{C}-6, \mathrm{C}-3$ and $\mathrm{C}-4$ respectively: all were observed due to natural abundance.

Initial signal assignment was made by comparison of ${ }^{13} \mathrm{C}$ chemical shifts with those of standard compounds recorded under comparable conditions. Additional information was obtained from further NMR experiments carried out on the perchloric acid extract. The DEPT method was used to establish the number of protons attached to each ${ }^{13} \mathrm{C}$ nucleus and this confirmed $\mathrm{CH}$ or $\mathrm{CH}_{2}$ for each signal in accordance with the assignments 
Table 1. Specific activities of various enzymes in strains AAG2 (pgi1 A) and S9 (pgi1 $\Delta$ spg29)

Strain S9 was grown to stationary phase in YEPD. Strain AAG2 was grown to stationary phase in YEPF, harvested and transferred to YEPD for $24 \mathrm{~h}$. The results are the means of duplicate determinations.

\begin{tabular}{|lrrr|}
\hline Enzyme* & $\begin{array}{c}\text { Specific activity } \\
\text { [mU (mg protein) }\end{array}$ & $\begin{array}{c}\text { Activity in } \\
\text { suppressed } \\
\text { strain }\end{array}$ \\
\cline { 2 - 3 } & AAG2 & S9 & $\begin{array}{c}\text { relative to } \\
\text { parent (\%) }\end{array}$ \\
\hline G6PDH & 206.5 & 194.3 & $94 \cdot 1$ \\
GND & 81.9 & $169 \cdot 1$ & 206.5 \\
RPE & 1.0 & 2.1 & $200 \cdot 7$ \\
TAL & 74.4 & 117.6 & 158.0 \\
NAD-GDH & 24.4 & 57.2 & 234.5 \\
NADP-GDH & 42.7 & 86.3 & 202.0 \\
\hline
\end{tabular}

* G6PDH, glucose-6-phosphate dehydrogenase; GND, 6-phosphogluconate dehydrogenase; RPE, ribulose-5-phosphate 3-epimerase; TAL, transaldolase; NAD-GDH, NAD-dependent glutamate dehydrogenase; NADP-GDH, NADP-dependent glutamate dehydrogenase.

given above. Further information was obtained from the ${ }^{13} \mathrm{C}-{ }^{1} \mathrm{H}$ correlation spectrum. In particular this confirmed that the ${ }^{13} \mathrm{C}$ at 65.3 is attached to protons at $\delta \mathrm{H} 3.47$ and $3 \cdot 55$, as expected for glycerol. The carbon signals attributed to ethanol C-2, glutamate C-4, glutamine C-4 and $\beta$-D-glucose C-1 were shown to be connected to protons having chemical shifts of $1.3,2.33,2.45$ and 4.6 respectively in accord with standard values.

\section{Enzyme assays on the pgi1 $\Delta$ parent and the pgi14 spg29 suppressed double mutant}

Phosphoglucose isomerase was undetectable in the parental strain AAG2 and in strain S9, confirming that both strains carry the pgi1 $\Delta$ mutation and that suppression in S9 was not due to the regaining of this activity. The specific activity of glucose-6-phosphate dehydrogenase was virtually identical in both strains, but the specific activities of all of the other enzymes assayed were all increased in the bypass mutant to $150-235 \%$ of the levels present in the parental strain (Table 1). The greatest increase in specific activity occurred in the case of NAD-dependent glutamate dehydrogenase.

\section{DISCUSSION}

Spontaneous mutants were isolated carrying mutations which suppress the pgi1 $\Delta$ mutation, thus allowing growth on glucose. In many respects these newly isolated spg mutants seem similar to those described by Aguilera (1987) and also the $\mathrm{rg} /$ mutants of Gamo et al. (1993). The reason for isolating these mutants was to establish the metabolic pathway used which enables a pgi1 $\Delta$ suppressed mutant to utilize glucose. ${ }^{13} \mathrm{C}$ NMR studies clearly showed that the hexose monophosphate pathway was used to suppress the defect in phosphoglucose isomerase. Despite the fact that previous studies all suggested the hexose monophosphate pathway was used in suppressed pgi1 strains (Aguilera, 1987; Gamo et al., 1993; Boles et al., 1993), this is the first time that the flux of carbon has actually been proved.

The specific activities of enzymes of the hexose monophosphate pathway were increased in the suppressed strain compared with the parental pgi14 strain with the exception of glucose-6-phosphate dehydrogenase. These results serve to confirm the NMR study and lead to the conclusion that glucose-6-phosphate dehydrogenase is not the limiting step of the metabolic bypass. Aguilera (1987) reported no significant differences in specific activities between pgi1s and pgi1 s spg1 double mutants. He also reported the specific activity of 6-phosphogluconate dehydrogenase as lower in the suppressed strains than in the pgi1 $\Delta$ parents whereas we observed that the specific activity of this enzyme was double compared with the parental strain. The reason for the difference between our results is likely to be because Aguilera grew both sorts of strain in YEP containing $2 \%$ fructose and $0.1 \%$ glucose, whilst we grew the pgi1d strain in this medium (it will not grow in YEPD), and then transferred it to YEPD for $24 \mathrm{~h}$ before determining enzyme activities; mutant S9 was grown in YEPD. The very reason for doing this was the possibility that the activity of one or more enzymes of the hexose monophosphate pathway might be higher in the presence of $(2 \%)$ glucose than $(2 \%)$ fructose.

The largest increase recorded for a single enzyme was for NAD-dependent glutamate dehydrogenase. This observation confirms the work of Boles et al. (1993) on the importance of this activity in suppressing pgi1 1 mutations. However, it is worth commenting that the elevation of NAD-dependent glutamate dehydrogenase is nothing like as high as the massive overexpression achieved by Boles $e t$ al. (1993), who put the GDH2 gene in a multi-copy plasmid. As demonstrated in the present study, S. cerevisiae does not have to achieve such a dramatic alteration to its normal metabolic activities: production of the large amounts of glycerol which were observed serves as an additional route for NAD regeneration. It is conceivable that other $s p g$ mutations result merely in the production of more glycerol without any increase whatsoever in the activity of NAD-dependent glutamate dehydrogenase, although it is not clear whether the extra glutamate produced (and not recycled to 2-oxoglutarate by NADdependent glutamate dehydrogenase) would be an advantage that allowed more rapid growth (glutamate is the major pool of nitrogen), or a detriment to the cell because of the depletion of an important intermediate of the tricarboxylic acid cycle.

In a study of carbon metabolism during sporulation in acetate it was noted that at a distinct time in the process metabolic flux around the hexose monophosphate pathway increased appreciably (Dickinson et al., 1983). Per- 
haps this up-regulation in sporulating diploids involves a gene which is mutated in $s p g$ mutants. Cloning of SPG genes will allow an examination whether the same genes are involved in controlling expression of hexose monophosphate pathway functions in sporulation and glycolytic bypass mutants.

\section{ACKNOWLEDGEMENTS}

This work was supported in part by grants to J.R.D. from the Royal Society, the British Council and United Distillers. M. A. S. was in receipt of an Earmarked Studentship to J.R. D. from the Biotechnology Directorate of the Science and Engineering Research Council.

\section{REFERENCES}

Aguilera, A. (1986). Deletion of the phosphoglucose isomerase structural gene makes growth and sporulation glucose dependent in Saccharomyces cerevisiae. Mol \& Gen Genet 204, 310-316.

Aguilera, A. (1987). Mutations suppressing the effects of a deletion of the phosphoglucose isomerase gene PGI1 in Saccharomyces cerevisiae. Curr Genet 11, 429-434.

Benevolensky, S. V., Clifton, D. \& Fraenkel, D. G. (1994). The effect of increased phosphoglucose isomerase on glucose metabolism in Saccharomyces cerevisiae. J Biol Chem 269, 4878-4882.

Boles, E., Lehnert, W. \& Zimmermann, F. K. (1993). The role of the NAD-dependent glutamate dehydrogenase in restoring growth on glucose of a Saccharomyces cerevisiae phosphoglucose isomerase mutant. Eur J Biochem 217, 469-477.

Breitenbach-Schmitt, I., Schmitt, H. D., Heinisch, F. \& Zimmermann, F. K. (1984). Genetic and physiological evidence for the existence of a second glycolytic pathway in yeast parallel to the phosphofructokinase-aldolase reaction sequence. Mol \& Gen Genet 195, 536-540.

Bruinenberg, P. M., Waslander, G. W., van Dijken, J.P. \& Scheffers, W. A. (1986). A comparative radiorespirometric study of glucose metabolism in yeasts. Yeast 2, 117-121.

Ciriacy, M. \& Breitenbach, I. (1979). Physiological effects of seven different blocks in glycolysis in Saccharomyces cerevisiae. J Bacteriol 139, 152-160.

Clifton, D., Weinstock, S. B. \& Fraenkel, D. G. (1978). Glycolysis mutants in Saccharomyces cerevisiae. Genetics 88, 1-11.

Davies, S. E. C. \& Brindle, K. M. (1992). Effects of overexpression of phosphofructokinase on glycolysis in the yeast Saccharomyces cerevisiae. Biocbemistry 31, 4729-4735.

Dickinson, J. R. (1991). Biochemical and genetic studies on the function of, and relationship between the PGI1- and CDC30encoded phosphoglucose isomerases in Saccharomyces cerevisiae. J Gen Microbiol 137, 765-770.

Dickinson, J. R. \& Hewlins, M. J. E. (1988). A study of the role of the hexose monophosphate pathway with respect to fatty acid biosynthesis in sporulation of Saccharomyces cerevisiae. J Gen Microbiol 134, 333-337.
Dickinson, J. R. \& Hewlins, M. J. E. (1991). ${ }^{13} \mathrm{C}$ NMR analysis of a developmental pathway mutation in Saccharomyces cerevisiae reveals a cell derepressed for succinate dehydrogenase. J Gen Microbiol 137, 1033-1037.

Dickinson, J. R. \& Williams, A. S. (1986). A genetic and biochemical analysis of the role of gluconeogenesis in sporulation of Saccharomyces cerevisiae. J Gen Microbiol 132, 2605-2610.

Dickinson, J. R., Dawes, I. W., Boyd, A. S. F. \& Baxter, R. L. (1983). ${ }^{13} \mathrm{C}$ NMR studies of acetate metabolism during sporulation of Saccharomyces cerevisiae. Proc Natl Acad Sci USA 80, 5847-5851.

Fraenkel, D. G. (1982). Carbohydrate metabolism. In The Molecular Biology of the Yeast Saccharomyces cerevisiae Life Cycle and Inberitance, pp. 1-37. Edited by J. N. Strathern, E. W. Jones \& J. R. Broach. Cold Spring Harbor: Cold Spring Harbor Laboratory.

Gamo, F.-J., Portillo, F. \& Gancedo, C. (1993). Characterization of mutations that overcome the toxic effect of glucose on phosphoglucose isomerase less strains of Saccharomyces cerevisiae. FEMS Microbiol Lett 106, 233-238.

Goffrini, P., Wéslowski-Louvel, M. \& Ferrero, I. (1991). A phosphoglucose isomerase gene is involved in the rag phenotype of the yeast Kluyveromyces lactis. Mol \& Gen Genet 228, 401-409.

Herrera, L. S. \& Pascual, C. (1978). Genetical and biochemical studies of glucosephosphate isomerase deficient mutants in Saccharomyces cerevisiae. J Gen Microbiol 108, 305--310.

Lloyd, D., James, C. J., Chapman, A. \& Dickinson, J. R. (1993). Combined ${ }^{13} \mathrm{C}$ NMR and mass-spectrometry for non-invasive monitoring of metabolism. Biotechnol Tech 7, 85-90.

London, R. E. (1988). ${ }^{13} \mathrm{C}$ labelling in studies of metabolic regulation. Progress in NMR Spectroscopy 20, 337-383.

Maitra, P. K. (1971). Glucose and fructose metabolism in a phosphoglucoisomeraseless mutant of Saccharomyces cerevisiae. J Bacteriol 107, 759-769.

Maitra, P. K. \& Lobo, Z. (1971). A kinetic study of glycolytic enzyme synthesis in yeast. $J$ Biol Chem 246, 475-488.

Mortimer, R. K. \& Hawthorne, D. C. (1975). Genetic mapping in yeast. Methods Cell Biol 11, 221-233.

Paquin, C. E. \& Williamson, V. M. (1986). Ty insertions at two loci account for most of the spontaneous antimycin A resistance mutations during growth at $15^{\circ} \mathrm{C}$ of Saccharomyces cerevisiae strains lacking ADH1. Mol Cell Biol 6, 70-79.

Sherman, F. (1975). Use of micromanipulators in yeast studies. Methods Cell Biol 11, 189-199.

Tchola, O. \& Horecker, B. L. (1966). Transaldolase. Methods Enzymol 9, 499-505.

Vinopal, R. T., Hillman, J. D., Schulman, H., Reznikoff, W. S. \& Fraenkel, D. G. (1975). New phosphoglucose isomerase mutants of Escherichia coli. J Bacteriol 122, 1172-1174.

Williamson, W. T. \& Wood, W. A. (1966). D-Ribulose-5-phosphate3-epimerase. Methods Envymol 9, 605-608.

Received 11 August 1994; revised 30 September 1994; accepted 11 October 1994. 